\title{
Towards In-baggage Suspicious Object Detection Using Commodity WiFi
}

\author{
Chen Wang*, Jian Liu*, Yingying Chen*, Hongbo Liu ${ }^{\dagger}$ and Yan Wang \\ *WINLAB, Rutgers University, New Brunswick, NJ, USA 08901 \\ Email: \{chenwang,jianliu\}@winlab.rutgers.edu, yingche@scarletmail.rutgers.edu \\ ${ }^{\dagger}$ Dept. of CIGT, IUPUI, Indianapolis, IN 46202 \\ Email: h145@iupui.edu \\ \#Dept. of CS, Binghamton University, Binghamton, NY 13902 \\ Email: yanwang@binghamton.edu
}

\begin{abstract}
The growing needs of public safety urgently require scalable and low-cost techniques on detecting dangerous objects (e.g., lethal weapons, homemade-bombs, explosive chemicals) hidden in baggage. Traditional baggage check involves either high manpower for manual examinations or expensive and specialized instruments, such as X-ray and CT. As such, many public places (i.e., museums and schools) that lack of strict security check are exposed to high risk. In this work, we propose to utilize the finegrained channel state information (CSI) from off-the-shelf WiFi to detect suspicious objects that are suspected to be dangerous (i.e., defined as any metal and liquid object) without penetrating into the user's privacy through physically opening the baggage. Our suspicious object detection system significantly reduces the deployment cost and is easy to set up in public venues. Towards this end, our system is realized by two major components: it first detects the existence of suspicious objects and identifies the dangerous material type based on the reconstructed CSI complex value (including both amplitude and phase information); it then determines the risk level of the object by examining the object's dimension (i.e., liquid volume and metal object's shape) based on the reconstructed CSI complex of the signals reflected by the object. Extensive experiments are conducted with 15 metal and liquid objects and 6 types of bags in a 6-month period. The results show that our system can detect over $95 \%$ suspicious objects in different types of bags and successfully identify $90 \%$ dangerous material types. In addition, our system can achieve the average errors of $16 \mathrm{ml}$ and $0.5 \mathrm{~cm}$ when estimating the volume of liquid and shape (i.e., width and height) of metal objects, respectively.
\end{abstract}

\section{INTRODUCTION}

The portable dangerous objects such as lethal weapons, homemade bombs, and explosive chemicals have posed an increasing threat to public security. In 2013, two homemade bombs detonated near the finish line of the annual Boston Marathon, causing 3 people dead and estimated 264 injured. In 2017, a gunman opened fire on a crowd of concertgoers at Harvest music festival on the Las Vegas Strip in Nevada, resulting in 58 people dead and 546 injured. In the above terrorist attacks, it is easy for the attackers to hide dangerous objects in small baggage without drawing any attention in public places. Due to the safety concerns following the recent shooting at a Florida high school, which left 17 people dead in April 2018, this high school now only allows the students to carry clear and transparent backpacks on campus [1]. But such measures also infringe the privacy of students, and may not be effective on preventing future attacks. To reduce such threats while preserving personal privacy, it is highly demanded of a wide deployment for non-intrusive security checks at the public places (e.g., museums, theme parks and schools).

Traditional in-baggage suspicious object detection involves either manual examination (e.g., setting up checkpoint at every entrance) or dedicated equipment (e.g., surveillance camera, X-ray machine, ultra-wide-band scanner) [2]-[4] and incurs high cost and deployment overhead, making them hard to scale. Recently, RF signals (e.g., WiFi and $60 \mathrm{GHz}$ radar) have shown their great potential in many non-intrusive sensing applications. For example, WiFi signals can be utilized to recognize human activities behind the wall [5] or perform coarse-grained imaging [6]. The $60 \mathrm{GHz}$ radar can be utilized to differentiate the objects (but cannot categorize the objects by material types) or perform imaging with two drones [7], [8]. However, these existing RF-based approaches involve high overhead by requiring a large antenna array or specialized signals. When a target object is placed in RF environments, both the object's inner (i.e., material content) and external (i.e., dimension and shape) properties contribute to the change of the wireless signals. Although the existing work can detect, track and image objects using RF signals, none of them separates the two influencing factors or applies them to finegrained sensing applications, such as material detection and shape imaging of the small objects in baggage.

Intuitively, most dangerous objects such as weapons, homemade bombs and explosives, are usually metal or liquid, which have significant interference (e.g., absorption, refraction and reflection) to wireless signals, while baggage is usually made of fiber, plastics or paper that allow wireless signals to pass through. Such different impacts to wireless signals suggest that it is possible to use wireless signals for detecting and identifying suspicious objects hidden in baggage. In this work, we leverage the fine-grained channel state information (CSI) that is readily available in low-cost WiFi devices to detect and identify suspicious objects hidden in baggage without intrusion (e.g., opening the bag). The basic idea is to examine the rich information of CSI complex, which includes both amplitude and phase information of wireless signals, to capture the various wireless interference caused by the materials and shapes of objects. Our system can be easily deployed to many places that still have no pre-installed security check infrastructures (e.g., airport) and require high-manpower to conduct security check such as theme parks, museums, stadiums, metro/train stations and scenic locations (e.g., Time Square). It uses the commodity WiFi to enable a low-cost and easy-to-scale solution, which provides the first-line of defense for detecting hidden suspicious objects. Our solution is timely as it demonstrates the possibility to reuse the prevalent WiFi technology to perform suspicious objects detection at every public area vulnerable to adversarial activities without 
introducing the high-cost security-checking infrastructure. In order to ensure that no dangerous item is carried through the entrances, our system requires to achieve low false negative rate of suspicious object detection. We focus on detecting the in-baggage suspicious objects defined as metal and liquid objects, which cover common dangerous items, and certain materials that could be confused with the dangerous items.

In particular, to identify different materials, we exploit the WiFi signals transmitting through or bypassing the object, which result in different characteristics (i.e., absorption, refraction and reflection) in the CSI complex values from antennas and their differences. Additionally, we extract the signal reflected by the object from CSI to estimate its shape (e.g., width and height) or volume based on the finding that the strength of the reflected signal is proportional to the reflection area of the object. Compared to existing work, our approach uniquely separates the wireless interference caused by two influencing factors of objects (i.e., material and shape) by exploiting different signal beams contained in the CSI complex. Our system only requires a WiFi device with 2 to 3 antennas and can be integrated into existing WiFi networks with low costs and deployment efforts, making it more scalable and practical than the approaches using dedicated instruments (e.g., X-ray and 60GHz radar).

A number of challenges need to be addressed to achieve the proposed system using off-the-shelf WiFi. First, the measured CSI from WiFi signals can be affected by a set of object's physical properties (e.g., material, shape, size and position), thus it is difficult to distinguish the different influences and identify the object's material and shape separately. Second, WiFi signals are not very suitable for object imaging due to its relative long wavelength comparing to the size of the target objects, which causes strong diffraction resulting in low imaging resolution. Third, detecting hidden objects in baggage needs to mitigate the effects of various types of bags. To address these challenges, we develop two system approaches specially designed for separating the refraction signals and the reflection signals from the CSI complex, and recognizing the object's material and shape, respectively. Our system eliminates the raw phase noise in CSI and reconstruct the CSI complex, which can robustly capture the dominant interference caused by material of suspicious objects even when the objects are hidden in the baggage. We also derive the reflection channel from CSI complex, which enables us to estimate the object's shape and volume at a finer level using the long-wavelength WiFi signals.

We summarize our main contributions as follows:

- We demonstrate that the readily available WiFi signals from low-cost devices can penetrate vision-blocked baggage and facilitate suspicious object detection and identification without dedicated devices or signals.

- We exploit the rich information in CSI complex to detect suspicious in-baggage objects and identify their categories (i.e., metal and liquid).

- We develop reflection-based risk level estimation method to determine the risk level of suspicious objects based on the estimated volume for liquid and the shape imaging for metal. We show that the pure reflection from the object can be extracted from the imperfect CSI (affected by unpredicted shift) in the $\mathrm{WiFi}$ device without requiring large antenna array or modifying the transmissions.
- Extensive experiments with 15 representative objects, 6 types of bags/boxes are conducted over a 6-month period. We show that our system can achieve over $95 \%$ and $90 \%$ accuracy for identifying the suspicious object and determining its material type and achieve an average error of $16 \mathrm{ml}$ and $0.5 \mathrm{~cm}$ for estimating liquid volume and metal object's shape.

\section{RELATED WORK}

Recently, there have been increasing security concerns at many public scenarios (e.g., security checkpoint of entrances) where object detection is urgently required. As traditional approaches, the vision-based techniques [2], [9] use infrared or regular cameras to identify objects according to their color, shape, texture, and temperature. These approaches, however, are sensitive to the environmental light intensity and either require a clear line-of-sight (LOS) between the object and cameras or require the target objects to have a relatively high temperature to be detected.

Moreover, a couple of studies adopt dedicated devices (e.g., [3], [7], [10]) to recognize target objects when the LOS is blocked. For instance, X-ray imagery [3] and CT volumetric imagery [10] have been used to obtain a $2 \mathrm{D}$ and $3 \mathrm{D}$ image of the baggage/parcel item for dangerous objects (e.g., firearms) detection, respectively. RadarCat [7] uses Frequency Modulated Continuous Wave (FMCW) radar operating in 60 $\mathrm{GHz}$ band to recognize different objects. Ultra-wide band phased array radar can also be used to image objects by seeing through the wall [4]. However, these approaches rely on expensive and specialized equipment, which do not facilitate the wide deployment in practice. Recently, RF-based sensing has drawn considerable attention. TagScan [11] deploys cheap RFID tags to identify the material type and image the horizontal profile of a target, but it requires a specialized tag reader, and it is not known whether it can be applied to in-baggage object detection. RF-Capture [12], [13] could capture the human figure (i.e., a coarse skeleton) leveraging the reflected RF signals through a wall with specialized devices, but it is dedicated for large human body and is questionable on identifying the materials of small objects. Dinesh et.al. [14] aims to utilize everyday commodity radios (i.e., smartphone) to detect and locate hidden objects leveraging the backscatter signal measurements, but it is hard to separate the influence of the object's material and size only from backscatter signal.

Due to the prevalence of WiFi devices, a recent study [6] explores the feasibility of achieving computational imaging by leveraging WiFi signals. The researchers operate Universal Software Radio Peripheral (USRP) at $2.4 \mathrm{GHz}$ band to image objects such as leather couches and metal shapes. But this method requires a large antenna array and is not sufficient to identify objects in a fine granularity manner, such as distinguishing the material of the objects. Furthermore, a set of studies use WiFi signals to sense minute human body movements to recognize/track human activities [5] and walking directions [15]. While these approaches mainly focus on exploiting the changes of fine-grained $\mathrm{WiFi}$ measurements (i.e., Channel State Information (CSI)) to sense human body movements, using WiFi signals to recognize small objects (e.g., water bottles, beverage cans, and knives) and different materials remains open. 


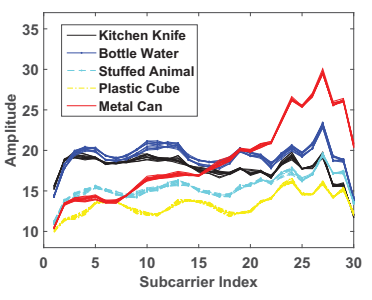

(a) Static objects' interference

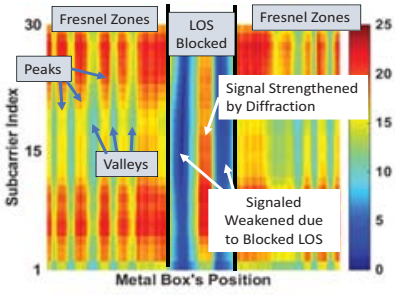

(b) Moving objects' interference
Fig. 1. Different objects' interference to the Wi-Fi signal (in CSI amplitude).

In this work, we conduct the first study to explore the feasibility of using low-cost off-the-shelf WiF devices to differentiate materials and types of the objects hidden in personal luggage or package boxes, which involves more challenges such as the different small objects in unknown positions of various bags or boxes. By exploring the rich context of CSI affected by the target object, we demonstrate that our approach can accurately estimate the inner nature (i.e., material) and outline properties (i.e., dimension/shape) of the hidden objects.

\section{Preliminaries \& System Design}

\section{A. Preliminaries}

Existing work has shown that the wireless channel of a stable WiFi environment could be easily changed by adding an object, for instance, a person, a bag or a cup. The intuition behind this is that interferences caused by the additional object, including absorption, reflection, and refraction of WiFi signals, largely change the multi-path effect of the existing WiFi environment and result in a different wireless channel. In this work, we find that such wireless channel changes caused by the additional object could be different due to different materials and shapes of the objects. To illustrate this intuition, we conduct some preliminary studies by respectively placing 5 common objects (i.e., a kitchen knife, a bottled water, a stuffed animal, a plastic cube, and a metal can) at the same position between a WiFi transmitter and a receiver that are one meter apart. Figure 1(a) presents the CSI amplitudes across 30 subcarriers corresponding to these objects. We can see that the CSI amplitude at each subcarrier is affected by the objects differently due to the object's different physical properties (e.g., material, size and shape). However, we find it is difficult to further distinguish the materials, shapes or sizes of different objects by examining the CSI amplitudes. Thus it is necessary to separate the wireless channel changes caused by objects' materials, shapes and sizes and explore more useful information from CSI in addition to its amplitude.

In addition, we notice that moving the object to multiple positions with a single-antenna setup can imitate the large antenna arrays [12], which could be exploited to perform object imaging. We illustrate this potential by conducting an experiment in which we move a metal box along a rail that is perpendicular to the line of sight (LOS) between a pair of single-antenna WiFi transmitter and receiver. Figure 1(b) shows the CSI amplitudes of 30 subcarriers collected while we move the metal box. We find that the metal box causes the strongest decrease in the amplitude when it blocks LOS, mainly because metal hardly let WiFi signals go through it. Such signal attenuation could be exploited to determine one dimension of the object (e.g., width or height). In addition, the repetitive peaks and valleys at all subcarriers on both sides of the LOS show the Fresnel Zones [16], which correspond to an object's reflection capability and can be utilized to estimate

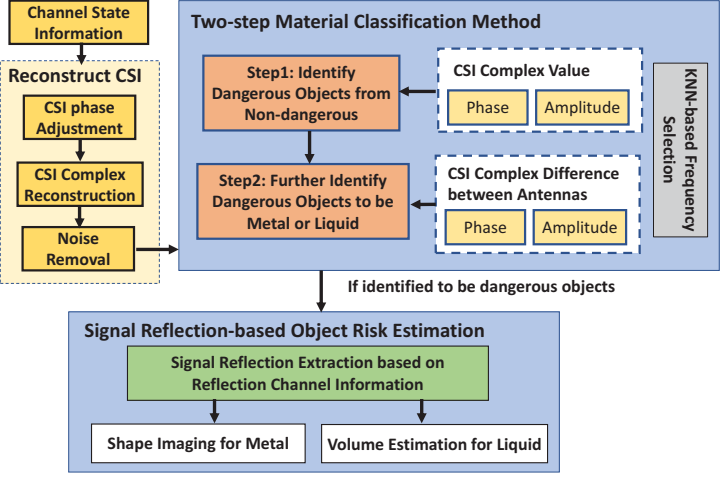

Fig. 2. System overview.

its' reflection surface area (related to both height and width). Ideally, we can estimate the dimension of an object by moving it crossing the LOS of a wireless channel like this. However, the strongest attenuation area due to the blocked LOS could be interfered by the diffraction of the WiFi signal at the small object (the strengthened signal in blocked LOS in Figure 1(b)). And estimating the dimension of an object directly using the peaks and valleys in Fresnel zone is not reliable because they are largely affected by the object's position and multi-path signals. Thus we need to seek solutions to extract the real reflection signal and reduce the influence of diffraction caused by the object to facilitate imaging the object.

\section{B. Threat Model}

Our work targets an adversary who intentionally or unintentionally carries dangerous items (e.g., lethal weapons, home- made bombs, combustibles) to public venues. Unlike tight security-checking areas (e.g., airports), there are two major types of areas vulnerable to adversarial activities: Places not having pre-installed security check infrastructures and employing high-manpower to perform security checks, such as theme parks, museums and stadiums, and the other kind even not having regulated checking process in place such as metro/train stations and scenic locations (e.g., Time Square). To launch an adversarial activity, the attacker usually hides the dangerous item in his bag or metal/plastic container to avoid being easily detected. In this work, we focus on detecting the suspicious objects including metal and liquid objects, which cover most of the dangerous objects that people could carry in baggage. More specifically, the metal objects such as aluminum cans, laptops, batteries and metal boxes can be used for homemade bombs, while the kitchen knives, guns and steel pipes can be directly used as weapons. Moreover, the liquids such as water, acid, alcohol and other chemicals in retainers might cause explosions.

\section{System Design}

System Requirements. Our system aims to automatically detect the suspicious objects in the aforementioned places. To achieve this goal, the design requirements of our system include: 1) A low false negative classification rate of suspicious objects in order to ensure adversaries cannot carry dangerous objects passing the security check; 2) A low system cost that is necessary to enable wide deployment at the places, which is lack of pre-installed security check infrastructures (e.g., museums, schools, stadiums, and train stations);3) Capability of identifying small objects that could be hidden in baggage; 4) Identifying both material and shape simultaneously. 


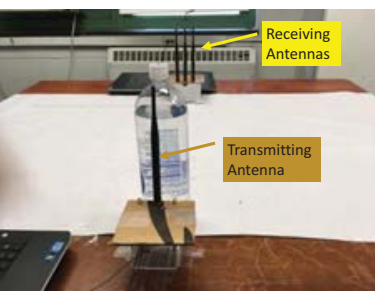

(a) Setup1: The Tx and Rx are placed apart to identify material

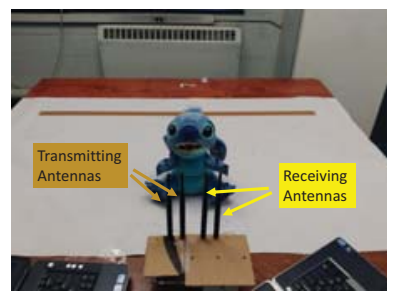

(b) Setup2: The Tx and Rx are placed closely to capture reflected signal
Fig. 3. Two experimental setups for object material identification and risk level estimation.

System Overview. To facilitate the suspicious object detection and identification, we design a novel system leveraging CSI measurements readily available in existing WiFi devices. As illustrated in Figure 2, our system takes the CSI from a pair of WiFi transmitter and receiver as input. The system then performs CSI Phase Adjustment and Complex CSI Reconstruction, which correct the CSI phase drifting and reconstruct the CSI complex including amplitude and corrected phase to describe the channel in an appropriate manner. Our system then performs Noise Removal to mitigate the interference of environmental noises. After that, the preprocessed CSI measurements would go through two main components: 1) Two-step Material Classification focuses on analyzing the material type to detect the suspicious objects in the black box while decreasing the influence factors including the object's size, shape and position; 2) Signal Reflection-based Object Risk Estimation can extract the reflected signal off the object from the CSI to perform shape imaging and volume estimation to estimate the risk level of the suspicious objects.

More specifically, Two-step Material Classification Method is performed to first identify existence of the suspicious objects by leveraging the CSI complex values and then derive the CSI complex difference between antennas to further distinguish the suspicious objects to be metal or liquid by capturing their minute differences. KNN-based Feature Selection is performed to select the good subcarriers for the CSI complex and CSI complex difference. Given the material identified, Signal Reflection-based Object Risk Estimation is performed to further estimate the suspicious object's risk level based on extracted reflections from the CSI complex. In particular, the object's risk level is determined by performing the shape imaging for the metal and the volume estimation for liquid in containers. This is because the liquid would have a higher risk level if its volume exceeds the permissible limit and metal piece is more suspicious if it has a similar shape to weapons.

Two WiFi-antenna Setups. Two uniquely setups (as shown in Figure 3) are designed for Material Classification and Object Risk Estimation respectively, by meeting the various requirements of the two different goals. When identifying the object's material, our system requires to focus only on the material influence on the CSI and reduce the influencing factors caused by the object's shape, size and position. In setup one (Figure 3(a)), the object is placed close to the transmitting antenna, while the receiving antenna is placed apart. By blocking much more spherical area of the transmitting signal, the object close to the antenna heavily affects the transmitting signals. Thus the signal beams passing through the object or bypassing the object' surface dominate the signal beams arriving at the receiver (except the multipath from permanent furniture), which are more related to the

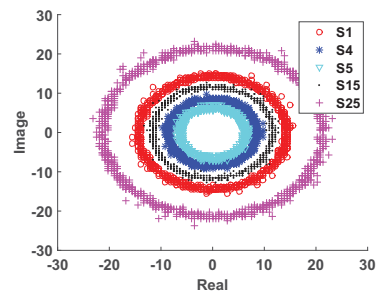

(a) Raw CSI complex value

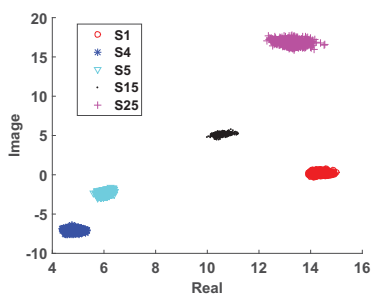

(b) Reconstructed CSI complex value
Fig. 4. The CSI before and after phase adjustment in the complex plane.

object's material influence. Moreover, due to the transmitting antenna's small elevation angle (e.g., 40 degree for 6dbi omniantenna), the signals are more focused to a small area on the object, which reduces the influence caused by object's size and shape. Additionally, the object blocks more inner Fresnel zones near the transmitter [16], which further weakens the arriving diffraction and reflection signals and reduce the influence of sizes, shapes and positions. Thus we can focuses on the object's material influence to CSI. Differently, the setup two (Figure 3(b)) amplifies the influence caused by the object's shape and size by placing the object away from the closely settled transmitter and receiver. It is good for imaging object's front face based on reflection and avoid the reflection from the short object's upper face. Note that these two setups can be combined in practical scenarios. For example, we can deploy two WiFi device pairs along a conveyor belt in most entrance check points to facilitate material identification and shape imaging in sequence automatically.

\section{CSI COMPleX VAlue ReCONSTRUCtion}

To facilitate the object detection and identification leveraging WiFi signals, we exploit CSI, the fine-grained description of the wireless channel, to capture the minute differences of the channel state change introduced by different objects. Specifically, the CSI with respect to each subcarrier is expressed as a complex value as follows:

$$
H\left(f_{k}\right)=\left|H\left(f_{k}\right)\right| e^{j<H\left(f_{k}\right)},
$$

where $H\left(f_{k}\right)$ describes the channel response for the subcarrier with central frequency $f_{k},\left|H\left(f_{k}\right)\right|$ and $\angle H\left(f_{k}\right)$ denote the corresponding amplitude and phase, respectively. It describes how the signal propagation is affected and reveals the impact of multipath effects between a pair of transceivers. The wireless channel will experience various impacts such as absorption, reflection and refraction by any object in the surrounding wireless environment, resulting in the changes of the CSI amplitude and phase at each subcarrier. However, the raw CSI extracted from WiFi signals could be distorted by the unpredicted phase shift and time lag caused by the nonsynchronized transmitter and receiver [17]. Most studies thus only use the CSI amplitude instead of the complex CSI value to characterize the wireless channel. Figure 4(a) shows the raw CSI complex values for 5 randomly chosen subcarriers across 1000 packets. We find that the raw CSI complex show the "doughnut" shape for each subcarrier because their amplitudes keep constant but the phases are much random. Thus the CSI phase needs to be adjusted for a more accurate description of the wireless channel.

Existing studies utilize the phase difference between adjacent subcarriers [18] or antennas [19] to remove the unknown phase shift, which may lose some useful information from the original CSI phase. In this work, we adopt the phase 

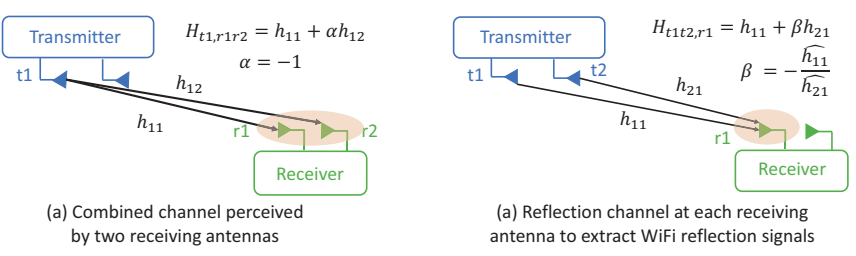

Fig. 5. Combined channel and reflection channel.

unwrapping [20] and the linear transformation method (similar to [17]) to adjust the raw CSI phase. In particular, we first unwrap the raw phase across all the subcarriers of each packet, which is wrapped within the range $[-p i, p i]$. Then a linear transformation is applied to the unwrapped phase to remove the phase shift offset at each subcarrier and thereby derive the adjusted phase $\angle \hat{H}\left(f_{k}\right)$ as:

$$
\left\{\begin{array}{l}
b=\frac{\angle H\left(f_{30}\right)-\angle H\left(f_{1}\right)}{f_{30}-f_{1}}, \\
a=\frac{1}{30} \sum_{k=1}^{30} \angle H\left(f_{k}\right), \\
\angle \hat{H}\left(f_{k}\right)=\angle H\left(f_{k}\right)-b f_{k}-a
\end{array}\right.
$$

where $k, k=1,2, \ldots, 30$ is the index of the 30 subcarriers and $f_{k}, f_{k}=-28,-26, \ldots, 28$ is the frequency point index of the real OFDM subcarrier [21](Table 7-25f).

Given the adjusted CSI phase, we reconstruct the complex form of CSI as $\hat{H}\left(f_{k}\right)=\left|H\left(f_{k}\right)\right| e^{j\left\langle\hat{H}\left(f_{k}\right)\right.}$, where $\angle \hat{H}\left(f_{k}\right)$ is the adjusted CSI phase. The reconstructed CSI complex $\hat{H}\left(f_{k}\right)$ accurately depicts the frequency response of each subcarrier in term of both amplitude and phase as shown in Figure 4(b), where the CSI complex of different subcarriers form their respective clusters in the complex plane. In a static wireless environment, both the CSI phase and amplitude maintain constant accordingly, which thus facilitates our two major system components to analyze the channel state changes introduced by the target objects with different materials, shapes and sizes.

\section{Two-step Material Classification based on CSI Complex VAlue}

In this section, we focus on the materials identification with our two-step method with the reconstructed CSI complex in Section IV, because the material (i.e., metal, liquid and unsuspicious) directly reflects whether the target object is suspiciously dangerous or not. The basic idea is to capture the wireless channel differences caused by different materials of target objects leveraging the CSI information. Different materials have different attributes on absorbing and refracting the WiFi signal, and such differences are reflected as the changes on CSI measurements. For example, 1) paper, cloth and plastics allow large portion of signal to penetrate; 2) the metal objects reflect a large portion of wireless signal and have the rest of signal scattered along its surface; 3 ) the liquid such as water has medium reflection but in the meanwhile allow a portion of signal to pass through.

\section{A. Examining the Material's Impact on Channel State}

We first examine how different materials influence the CSI complex. Figure 6 (a) shows the CSI complex values with respect to one subcarrier with 9 different objects in Setup One (Figure 3(a)), where each object was tested three times with slight position and orientation changes. We can observe that the suspicious objects such as metal and water have their CSIs clustered together. In comparison, the CSIs corresponding to other objects such as fiber, books and plastics form another different cluster overlapped with the cluster

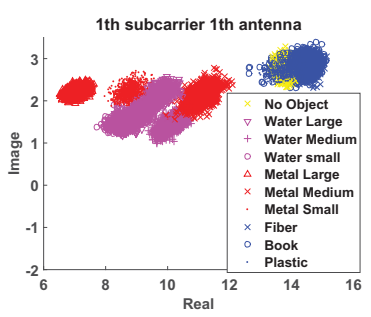

(a) Differentiate dangerous objects from non-dangerous based on CSI

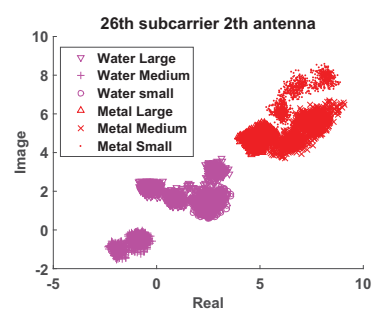

(b) Differentiate metal from liquid based on CSI difference
Fig. 6. Two-step material identification method based on CSI complex and CSI complex difference.

when there is no object present (i.e., yellow dots). This is because these unsuspicious objects have little interference to the wireless channel due to their electric-insulated attributes and low density. Moreover, the metal objects and the water containers of different sizes are all significantly different from the unsuspicious objects in term of CSI complex. Therefore, regardless of the sizes and shapes, the suspicious objects can be distinguished effectively from the unsuspicious objects based on the reconstructed CSI complex. Note that the most bags/boxes showing at the theme park, museum entrance are made of the non-dangerous material such as fiber, paper and plastics, and thus they have little impact to the wireless channel. Accordingly, the hidden suspicious objects could dominate the interference to CSI complex and be easily detected.

\section{B. CSI Complex Difference between Receiving Antennas}

With the capability to tell suspicious materials from unsuspicious ones, the CSI complex alone is still hard to further distinguish the different types of suspicious materials. For example, as shown in Figure 6(a), the CSI clusters corresponding to liquid and metal objects are close to each other. This is because these suspicious materials all heavily interfere the wireless channel. Thus we need to further distinguish their minute difference by resorting to more in-depth information such as the relative spatial information from multiple antennas. For example, different materials have different scattering effects on the RF signals when passing through the object. Therefore, we propose to leverage the CSI complex differences between any two receiving antennas to capture the minute difference of the signal scattering at multiple antennas. Assuming that the transmitter emits a symbol $x$ at antenna $t 1$, the symbols received by the two antennas $r 1$ and $r 2$ of the receiver would be $h_{11} x$ and $h_{12} x$ (as shown in Figure 5(a)), where $h_{11}$ and $h_{12}$ are the CSI for the $t 1-r 1$ and the $t 1-r 2$ antenna pair. Then the combined input y1 at the two receiving antennas could be defined as $y 1=\left(h_{11}+\alpha h_{12}\right) x$. By choosing $\alpha=-1$, we define the combined channel $H_{t 1, r 1 r 2}$ between $\mathrm{t} 1$ and $\mathrm{r} 1, \mathrm{r} 2$ as,

$$
H_{t 1, r 1 r 2}=h_{11}-h_{12}
$$

Under the presence of an object, the combined channel $H_{t 1, r 1 r 2}$ measures the difference between the two channel states, which removes the common factors (e.g., permanent furniture influence) at two receiving antennas, and also amplifies the minute differences on scattering effects caused by different materials. As illustrated in Figure 6(b), the metal and water could be differentiated by the CSI complex difference regardless of their sizes. We then utilize the CSI complex difference to identify the types of suspicious materials.

\section{Two-step Method Implementation}

Based on the above observations, we develop a two-step material identification method to classify the object's material 


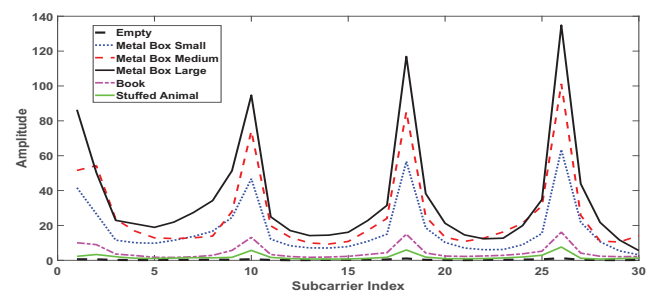

Fig. 7. The reflection channel state information in response to different objects' reflections.

within Setup One. In particular, 1) we first differentiate the suspicious objects from unsuspicious ones by leveraging the reconstructed CSI complex values as features to perform classification; 2) we next identify whether the material of the dangerous objects is metal or liquid by deriving the CSI complex differences between two receiving antennas as the features for further categorization. At each step, we apply a learning-based method to build the material profiles. During the training phase, we first apply the KNN-based feature selection method to choose CSI-based features from good subcarriers and antenna pairs. In particular, we cluster the CSI-based features with respect to each subcarrier based on $\mathrm{KNN}$; then k-fold cross validation is applied to the KNN-based clusters to determine the good subcarriers and antenna pairs which show lower K-fold loss ratio than a predefined threshold when differentiating the materials at each step. Next, a learning method, such as SVM or deep learning, is adopted to train the material profile at each step. Note that, to identify the object within different baggage, we pick several representative types of bags/boxes with the target objects enclosed to build the CSI profiles. During the testing process, the CSI and CSI complex difference of target objects are compared with the pre-defined profiles for classification. As long as their material belongs to the three types (i.e., metal, liquid and unsuspicious), our system can identify them accurately. Moreover, most bags/boxes are made of unsuspicious material such as fiber, the hidden dangerous objects, if any, could dominate the impact on the CSI, which can be easily captured by our proposed system. Therefore our system can differentiate the materials of hidden target objects wrapped by various bags/boxes.

\section{OBJECT Risk ESTIMATION LEVERAGing Signal REFLECTION-BASED OBJECT IMAGING}

It is not sufficient to determine the risk of the suspicious objects by identifying the material only. For instance, the volume of the liquid less than a certain limit (e.g., 100ml) is less risky and is usually allowed to be carried on flights; the metal pieces with similar shapes as the weapons (e.g., kitchen knife and soda-can bomb) are usually more dangerous. WiFi signals from off-the-shelf devices are not specifically designed for the small object imaging due to its long wavelength (e.g., $12 \mathrm{~cm}$ for $2.4 \mathrm{GHz}$ and $6 \mathrm{~cm}$ for $5 \mathrm{Gz}$ ), which would induce strong diffraction and thereby significantly decrease the imaging resolution [8]. To mitigate the effects of signal diffraction for better imaging resolution, we focus on the signals reflected from the target object to perform metal object imaging and liquid volume estimation.

\section{A. Extracting Reflected Signals from CSI Complex}

We first introduce how to extract the signal reflected by the target object from the CSI complex based on Setup Two (i.e., Figure 3(b)). As shown in Figure 5(b), two transmitting antennas (i.e., $t 1$ and $t 2$ ) and one of the receiving antennas

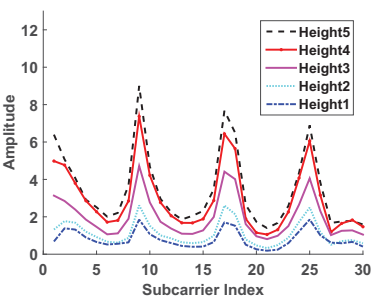

(a) Increasing liquid height in the same container (b) Increasing liquid height in the containers of different sizes

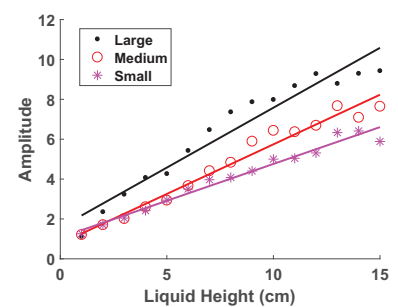

Fig. 8. CSI amplitude changes with increasing volume of liquid.

(e.g., ri) are considered for illustration. The channel response capturing the signals reflected from the target object only, defined as Reflection Channel $H_{t 1 t 2, r i}$, can be represented as:

$$
H_{t 1 t 2, r i}=h_{1 i}+\beta h_{2 i}, \quad \beta=-\frac{\hat{h}_{1 i}}{\hat{h}_{2 i}},
$$

where $h_{1 i}$ and $h_{2 i}$ are the estimated channel states (i.e., CSI) for two antenna pairs (i.e., from transmitting antenna $t 1$ and $t 2$ to receiving antenna $r i$ respectively). The weight $\beta=-\frac{\hat{h}_{1 i}}{\hat{h}_{2 i}}$ is calculated by $\hat{h}_{1 i}$ and $\hat{h}_{2 i}$, which are the channel states with no target object presented in the area of interest. When no object is placed, the signals from the transmitting antenna $t 1$ and $t 2$ are combined linearly to null the reflection paths to the receiving antenna $r i$. Therefore the LOS and the reflected paths from the permanent furniture [22] are eliminated in the channel state information. But when an object is placed in the area, the reflected paths will become in-negligible, and the amplitude of reflected channel information $H_{t 1 t 2, r i}$ implies the object's reflecting capability. Figure 7 shows an example of the amplitudes of the reflected channel state information (reflection CSI) perceived by receiving antenna $r 1$ with different objects presented. In particular, empty environment renders close to zero amplitude for all subcarriers of $H_{t 1 t 2, r i}$ amplitudes (i.e., black dash line), whereas the unsuspicious objects such as book and stuffed animal result in none zero amplitudes but much lower than metal objects. Moreover, we also find the sizes of the metal objects are proportional to the reflected CSI amplitudes of all subcarriers, and different subcarriers also have different sensitivity when they are reflected from the objects. The above observations confirm the effectiveness of our proposed method on capturing the signals reflected from target objects by eliminating the LOS and multipath signals. We next leverage the captured reflected signals to estimate the liquid volume and perform metal object imaging.

\section{B. Volume Estimation for Liquid Objects in Baggage}

To estimate the liquid volume, we conduct some experiments under Setup Two (i.e., Figure 3(b)), which involves a small bottle as the target object with 5 different water volumes ranging from empty to full. The amplitudes of the reflected CSI (i.e., $H_{t 1 t 2, r 1}$ ) corresponding to different water volumes are shown in Figure 8(a). It is easy to find that the larger the water volume, the greater the reflected CSI amplitude across all 30 subcarriers due to the increasing reflecting surface. To further quantify the relationship between the water volume and the amplitude of reflection CSI, we select 15 different water heights in three cylindrical containers of different diameters (i.e., large, medium and small). As shown in Figure 8(b), we observe that the amplitude of the reflected CSI is linearly proportional to the water heights for all three containers. Moreover, the larger container has faster 


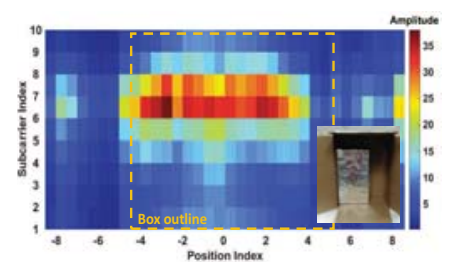

(a) Tinfoil box in a package box

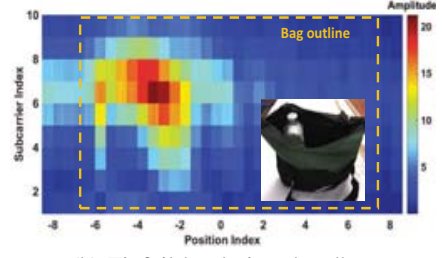

(b) Tinfoil bottle in a handbag

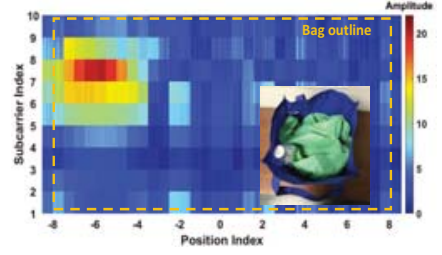

(c) Tinfoil bottle in a tote bag stuffed with clothes

Fig. 9. The amplitude of reflection channel state information in response to the metal objects in a baggage.

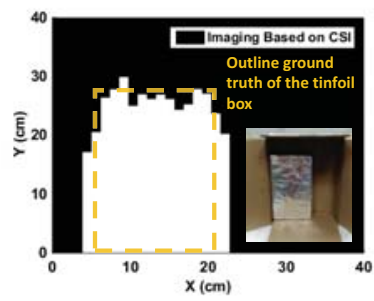

(a) Tinfoil box in a package box

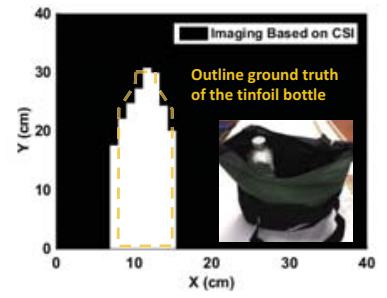

(b) Tinfoil bottle in a handbag

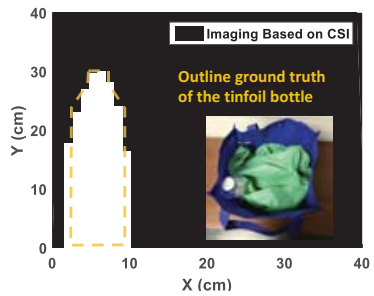

(c) Tinfoil bottle in a tote bag stuffed with clothes

Fig. 10. Using the WiFi reflections extracted from CSI to image the metal objects in baggage.

growth rate on the CSI amplitude due to the larger reflecting surface under the same water height. Therefore, as long as the container's diameter is determined, the liquid's volume can be derived by following a linear regression model. In this work, we assume the liquid is kept in the nonmetal cylindrical containers such as plastic or glass bottle. If the liquid is in metal containers, it would be identified as metal objects based on our material identification method in Section V.

Based on our preliminary study, the liquid volume estimation consists of two steps, diameter determination and liquid height estimation. To determine the diameter of the liquid container, we adopt the same method of determining the metal object's width as in Section VI-C. Once the liquid container diameter is obtained, we apply two different methods, the linear regression method and the neural network-based method, to estimate the liquid height by leveraging the frequency selection property across multiple subcarriers. Specifically, the linear regression method aims to build the linear regression relationship between the CSI amplitude and liquid height for each subcarrier, and integrate the prediction results from all subcarriers to derive the liquid height. The neural networkbased method predict the unknown height of the liquid in containers by building a neural network model, which takes the amplitudes of all subcarriers with respect to different liquid heights as the training feature vector. At last, the liquid volume is easily obtained based on the estimated container diameter and the liquid height.

\section{Shape Imaging for Metal Objects in Baggage}

Unlike the existing studies relying on large antenna arrays to determine the shape of metal objects, we propose to image the in-baggage metal objects using commercial WiFi devices with a limited number of antennas while the baggage is moved by the conveyor belt, which is available at many entrance check points. Figure 9 shows the reflection channel response $H_{t 1 t 2, r 1}$ when the target object is in an opaque baggage, which moves along the track in parallel with the antenna array. The rectangular box and the water bottle are covered with tinfoil to imitate the metal objects of different shapes that are similar to homemade bombs. We find that the reflected channel response is greater when the target object is close to the central line between the transmitter and receiver, where strong reflection is usually incurred by the object. Moreover, as shown in Figure 9(a) and (b), both the width and position of the target object hidden in the baggage or box can be clearly identified from the reflected CSI amplitude (e.g., red color). Furthermore, when there are multiple objects in the same baggage, such as the metal object together with clothes as shown in Figure 9(c), the metal object dominates the reflection signals and can still be distinguished and imaged. Note that our system can detect the existence of suspicious objects even if liquid and metal objects are in the same baggage and the object imaging includes both objects. We therefore develop a threshold-based approach to capture the outline of the metal objects and separate them from other nonsuspicious objects, including the baggage. We first estimate object's width, which is proportional to the object moving distance that cause reflections above a threshold by using $d=\gamma \hat{d}$, where $\hat{d}$ is the estimated width from reflection CSI amplitude and $\gamma$ is the ratio, which is related to the short wavelength of WiFi signal. Once the width of the object is determined, we proceed to estimate the object's height based on the fact that the reflection CSI amplitude is proportional to the reflection area. The estimation of the metal object's height is similar to the method in Section VI-B. Figure 10 shows the final imaging results of the metal objects based on the reflection CSI amplitude of Figure 9. It is encouraging to find that the metal object's outlines can be well recognized, which are very close to the actual shape of the target objects even when it is hidden with other objects in the baggage.

\section{A. Experimental Methodology}

ViI. Performance Evaluation

Experimental Setup. We implement our system on a pair of laptops, which are equipped with IWL 5300 wireless cards and three $6 \mathrm{dBi}$ omnidirectional dual band rubber ducky antennas. The two laptops are placed upon a wooden table in a typical indoor room, and we employ two setups as shown in Figure 3 to perform material identification and risk level estimation, respectively. The laptops are running Ubuntu 10.04 LTS with the kernel 2.6.36, and the WiFi card works at $5 \mathrm{GHz}$ frequency band with the transmission rate $100 \mathrm{pkt} / \mathrm{sec}$. During data collection, two people are in the room standing by the table to imitate the practical scenarios.

Target Objects. We evaluate our system with the combination of 15 different target objects in three categories (i.e. metal, 


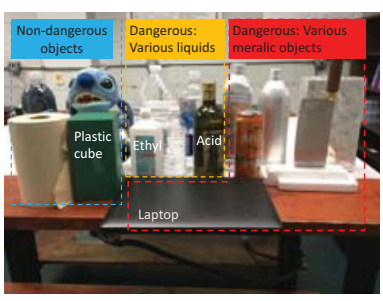

(a) Fifteen objects in 3 categories

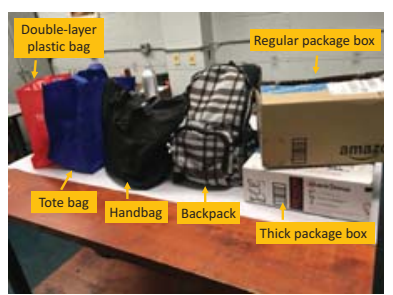

(b) Six different bags and boxes

Fig. 11. Various target objects and bags/boxes in the experiment.

liquid and non-dangerous) and 6 representative bags/boxes in three categories (i.e., backpack/handbag, cardboard boxes, thick plastic bag) as shown in Figure 11. For the material identification, we put each of the 15 objects in 6 bags/boxes respectively and experiment under Setup1 in Figure 3. Each experiment is repeated 5 times while slightly changing the object's position and orientation. For dangerous object risk level estimation, we place the metal objects across multiple positions under Setup2 (i.e., Figure 3(b)) to estimate the size (i.e. width and height). Moreover, we have the three different size containers (i.e., large, medium and small) filled with different volumes of liquid to estimate liquid volume. Overall, over 800 experimental data traces are collected during a 6month period to evaluate our proposed system.

Evaluation Metrics. To evaluate the material identification method, we defineIdentification Accuracy as the ratio of the correctly identified objects over all the tested objects, and define Detection Rate as the ratio of correctly identified objects over the total objects of the same material. A high detection rate of the suspicious object reflects a low false negative rate, which guarantees that few suspicious objects could pass the security check. To evaluate the risk level estimation, we utilize Size Estimation Error $(\mathrm{cm})$ to measure the estimation of the metal object' width and height and Volume Estimation Error $(\mathrm{ml})$ for the estimation of the liquid volume.

\section{B. Material Classification}

We first evaluate our material identification of the object hidden in various bags, especially when different number of bags are used for training the profile. Figure 12 shows that our system can achieve high accuracy in identifying the object's material when they are put in different bags. In particular, given the combination of all the 15 objects and the 6 bags in our profile, Figure 12(a) shows that our system can achieve 99\% accuracy in classifying dangerous objects from nondangerous (step1) and 97\% accuracy to further differentiate the dangerous objects to be metal and liquid (step2). Figure 12(b) further shows that the overall detection rate for the dangerous material, metal and liquid are 99\%, 98\% and 95\%. Moreover, we find that the material identification accuracy reduces a little bit as the number of bags used for profile training decreases. For example, when using half of the bags (i.e., one bag/box from each of three categories) for training, the step1 and step2 accuracy of our material classification method fall to $95 \%$ and $90 \%$ while the detection rate of dangerous objects decreases to $94 \%$. The overall detection rate for metal and liquid objects fall to $90 \%$ and $92 \%$. This is because the bags and boxes, though made of non-dangerous material, still induce slightly different interferences on the wireless channel, thereby resulting in the errors in material detection. But because the bags used in testing phase have the similar material with the bags/boxes used in building training profile,

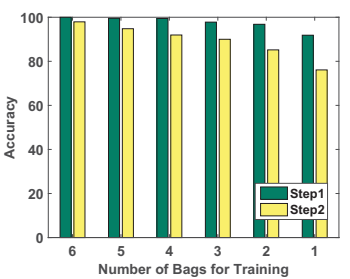

(a) Accuracy for two material identification steps

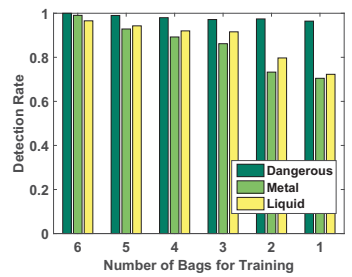

(b) Detection rate for the various objects
Fig. 12. Material identification with different number of baggage in profile.

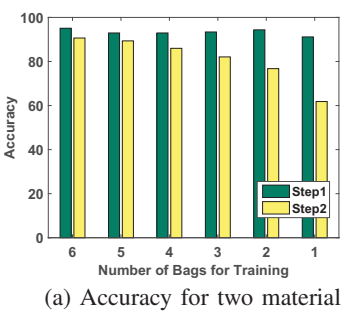

identification steps

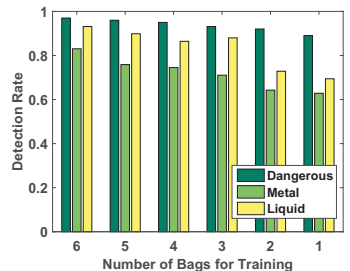

(b) Detection rate for the various objects
Fig. 13. Material identification with half objects and different number of baggage in profile.

our system still achieves high material identification accuracy. Additionally, regardless of the number of bags used in training phase, our system can keep over 93\% accuracy of detecting the dangerous material as shown in Figure 12(b).

Figure 13 presents a more challenging scenario, where only half of the objects in each of the three object categories are trained to build the profile. Figure 13(a) shows that in this scenario, if all the bags are used for training, we can achieve over $95 \%$ accuracy for step 1 and $90 \%$ for step 2 . The overall detection rate for the dangerous materials is $96 \%$, and the detection rate for metal and liquid objects fall to $82 \%$ and 91\% as shown in Figure 13(b). Furthermore, we find that the material identification accuracy also reduces with decreasing number of bags used for training, due to the different bags' slight different interference. In particular, when half of the objects and half of the bags are used for training the profile, our system can achieve $91 \%$ and $85 \%$ accuracy for step 1 and step2 of our material classification and the detection rates for the dangerous, metal and liquid are around 90\%, 78\% and $85 \%$. The results show that our system can efficiently identify the object made of dangerous material and further classify the dangerous material types in the more complex scenarios. In an extreme case, when half of the objects and only one bag are chosen for training, the detection rate for all dangerous materials is still over $89 \%$. The results confirm that our system can efficiently recognize the object by its material regardless of their shapes and sizes or what bags they are hidden in.

\section{Risk Level Estimation based on Object Imaging}

We next evaluate the performance of our system on estimating the risk level of the objects through object imaging (i.e., metal object size and liquid volume).

Metal Object Size Estimation. Figure 14(a) shows the results of our system on estimating the sizes of different metal objects. We find that our system can achieve cm-level accuracy on the size estimation of metal objects. In particular, over $80 \%$ estimation error of the metal object's widths and heights are within $0.7 \mathrm{~cm}$ and $90 \%$ within $1 \mathrm{~cm}$. The average errors for estimating the metal object's width and height are $0.3 \mathrm{~cm}$ and $0.5 \mathrm{~cm}$, respectively. The results show that our system can 


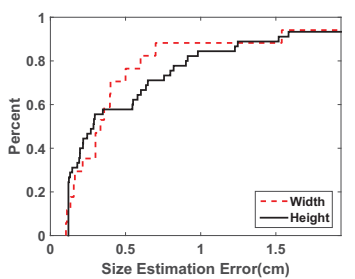

(a) Metal objects' width and height

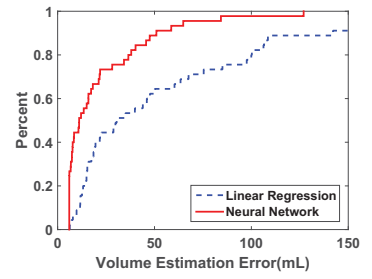

(b) Liquid volume estimation
Fig. 14. Accuracy of estimating the object's size and volume.

estimate the metal objects' size accurately, which is good to perform accurate object imaging and infer whether the metal object is suspicious to be deadly weapons or bombs.

Liquid Volume Estimation. The performance of liquid volume estimation is presented in Figure 14(b), where we apply two different methods, linear regression and neural network for the volume estimation respectively. We find that both methods can achieve high accuracy on liquid volume estimation. The neural network-based method achieves even higher accuracy with the median error as small as $16 \mathrm{ml}$. Moreover, over $80 \%$ estimation errors are within $35 \mathrm{ml}$. The results validate that our system can accurately estimate the liquid volume, and provide significant information to derive the risk level of liquid objects.

\section{CONCLUSION}

This paper explores the feasibility of using off-the-shelf WiFi signals to detect suspicious objects (i.e., metal and liquid objects) hidden in baggage without penetrating into the user's privacy. Our solution is timely as it demonstrates the possibility to reuse the prevalent WiFi technology to perform suspicious objects detection at every public area vulnerable to adversarial activities without requiring the installation of high-cost security-checking infrastructures. The designed system can also estimate the risk level of the target object through object imaging to estimate the shape/volume of the metal/liquid objects. Specifically, we deploy two different system setups for separating the refraction signals and the reflection signals from the CSI complex and recognizing the object's material and shape, respectively. Our system removes the raw phase noise in CSI and reconstructs the CSI complex, which can robustly capture the dominant interference caused by the suspicious material even when the object is hidden in the baggage. We also derive the reflection channel from CSI complex that can enable us to estimate the object's shape and volume at a fine level using the long-wavelength WiFi signals. Extensive experiments are conducted with 15 objects and 6 bags over a 6-month period. The results show that our system can detect over $95 \%$ dangerous objects in different types of bags and successfully identify $90 \%$ dangerous material types. In addition, our system can achieve the average errors of $16 \mathrm{ml}$ and $0.5 \mathrm{~cm}$ when estimating the shape/volume of the metal/liquid object, respectively.

Acknowledgments. This work is supported in part by the NSF grant CNS1820624 and Army Research Office W911NF17-1-0467.

\section{REFERENCES}

[1] P. MAZZEI, "Florida school, on edge since shooting, requires students to carry clear backpacks," https://www.nytimes.com/ 2018/03/21/us/florida-school-shooting-clear-backpacks.html, 2018.

[2] S. Chi and C. H. Caldas, "Automated object identification using optical video cameras on construction sites," Computer-Aided Civil and Infrastructure Engineering, vol. 26, no. 5, pp. 368-380, 2011.
[3] D. Turcsany, A. Mouton, and T. P. Breckon, "Improving featurebased object recognition for x-ray baggage security screening using primed visualwords," in IEEE International Conference on Industrial Technology, 2013, pp. 1140-1145.

[4] T. S. Ralston, G. L. Charvat, and J. E. Peabody, "Real-time throughwall imaging using an ultrawideband multiple-input multiple-output (mimo) phased array radar system," in IEEE International Symposium on Phased Array Systems and Technology, 2010, pp. 551-558.

[5] Y. Wang, J. Liu, Y. Chen, M. Gruteser, J. Yang, and H. Liu, "E-eyes: device-free location-oriented activity identification using fine-grained wifi signatures," in Proceedings of the 20th annual international conference on Mobile computing and networking (ACM MobiCom), 2014, pp. 617-628.

[6] D. Huang, R. Nandakumar, and S. Gollakota, "Feasibility and limits of wi-fi imaging," in Proceedings of the 12th ACM Conference on Embedded Network Sensor Systems, 2014, pp. 266-279.

[7] H.-S. Yeo, G. Flamich, P. Schrempf, D. Harris-Birtill, and A. Quigley, "Radarcat: Radar categorization for input \& interaction," in Proceedings of the 29th ACM Annual Symposium on User Interface Software and Technology, 2016, pp. 833-841.

[8] Y. Zhu, Y. Zhu, Z. Zhang, B. Y. Zhao, and H. Zheng, "60ghz mobile imaging radar," in Proceedings of the 16th ACM International Workshop on Mobile Computing Systems and Applications, 2015, pp. 75-80.

[9] X. Wang, "Intelligent multi-camera video surveillance: A review," Pattern recognition letters, vol. 34, no. 1, pp. 3-19, 2013.

[10] G. T. Flitton, T. P. Breckon, and N. M. Bouallagu, "Object recognition using $3 \mathrm{~d}$ sift in complex ct volumes," in Proceedings of the British Machine Vision Conference, 2010, pp. 1-12.

[11] J. Wang, J. Xiong, X. Chen, H. Jiang, R. K. Balan, and D. Fang, "Tagscan: Simultaneous target imaging and material identification with commodity rfid devices," in Proceedings of the 23rd Annual International Conference on Mobile Computing and Networking (ACM MobiCom), 2017, pp. 288-300.

[12] F. Adib and D. Katabi, "See through walls with wifi!" in Proceedings of the ACM SIGCOMM conference, 2013.

[13] F. Adib, C.-Y. Hsu, H. Mao, D. Katabi, and F. Durand, "Capturing the human figure through a wall," ACM Transactions on Graph., vol. 34, no. 6, pp. 219:1-219:13, 2015.

[14] D. Bharadia, K. R. Joshi, and S. Katti, "Full duplex backscatter," in Proceedings of the Twelfth ACM Workshop on Hot Topics in Networks, 2013.

[15] D. Wu, D. Zhang, C. Xu, Y. Wang, and H. Wang, "Widir: walking direction estimation using wireless signals," in Proceedings of the 2016 ACM International Joint Conference on Pervasive and Ubiquitous Computing. ACM, 2016, pp. 351-362.

[16] H. Wang, D. Zhang, J. Ma, Y. Wang, Y. Wang, D. Wu, T. Gu, and B. Xie, "Human respiration detection with commodity wifi devices: do user location and body orientation matter?" in Proceedings of the 2016 ACM International Joint Conference on Pervasive and Ubiquitous Computing. ACM, 2016, pp. 25-36.

[17] S. Sen, B. Radunovic, R. R. Choudhury, and T. Minka, "Spot localization using phy layer information," in Proceedings of the 10th international conference on Mobile systems, applications, and services (ACM MobiSys), 2012.

[18] C. Wang, X. Zheng, Y. Chen, and J. Yang, "Locating rogue access point using fine-grained channel information," IEEE Transactions on Mobile Computing, 2016.

[19] S. Kumar, S. Gil, D. Katabi, and D. Rus, "Accurate indoor localization with zero start-up cost," in Proceedings of the 20th annual international conference on Mobile computing and networking (ACM Mobicom), 2014, pp. 483-494.

[20] A. A. Hassan, W. E. Stark, J. E. Hershey, and S. Chennakeshu, "Cryptographic key agreement for mobile radio," Digital Signal Processing, vol. 6, no. 4, pp. 207-212, 1996.

[21] I. Std., "802.11n-2009: Enhancements for higher throughput," Available at http://www.ieee802.org, 2009.

[22] K. C.-J. Lin, S. Gollakota, and D. Katabi, "Random access heterogeneous mimo networks," in ACM SIGCOMM Computer Communication Review, vol. 41, no. 4, 2011, pp. 146-157. 